\title{
RESPIRATORY IRREGULARITY AND STRESS HORMONES IN PANIC DISORDER: EXPLORING POTENTIAL LINKAGES
}

\author{
James L. Abelson, M.D., Ph.D., ${ }^{*}$ Samir Khan, Ph.D., Mark Lyubkin, M.D., and Nicholas Giardino, Ph.D.
}

\begin{abstract}
Dysregulation within both respiratory control systems and the bypothalamicpituitary adrenal (HPA) axis has been implicated in the patbophysiological of panic disorder. However, potential linkages between respiration and the HPA axis have rarely been examined in panic patients. We have previously published neuroendocrine and psychophysiological response data from a laboratory panic model using the respiratory stimulant doxapram. We now present a new, theoretically driven re-examination of linkages between HPA axis and respiratory measures in this model. Previous analyses showed elevated corticotropin (ACTH) and persistent tidal volume irregularity in panic patients, due to a high frequency of sighs. Regression analyses now show that tidal volume irregularity and sigh frequency were strongly predicted by pre-challenge ACTH levels, but not by subjective distress or panic symptoms. We predicted this relationship on the basis of our hypothesis that both the HPA axis and respiratory control systems may be reactive to contextual cues such as novelty or anticipation of future challenge. Follow-up work is needed to directly test this bypothesis. Depression and Anxiety 25:885-887, 2008. $\quad$ Published 2007 Wiley-Liss, Inc. ${ }^{\dagger}$
\end{abstract}

Key words: corticotropin; respiration; breathing; panic disorder; stress

\section{INTRODUCTION}

Dysregulation within brainstem respiratory control systems may play a central role in the etiology or pathophysiology of panic disorder [Klein, 1993]. Considerable evidence in fact supports the presence of dysregulated respiratory control in panic patients [Papp et al., 1997]. Another etiologic theory suggests that panic disorder may be due to dysregulation within central stress response circuits mediated by corticoptropin-releasing hormone (CRH) [Schreiber et al., 1996]. There is evidence that panic disorder patients do have abnormalities within the hypothalamic-pituitary adrenal (HPA) axis, which is controlled by CRH [Abelson and Curtis, 1996]. We have made contributions to both sets of literature. We have shown that panic patients have persistent respiratory (tidal volume) irregularity, characterized by a high frequency of sighing breaths. This irregularity is relatively immutable even when respiration is driven exogenously; and it is unaffected by cognitive manipulation that significantly alters other respiratory measures as well as panic attack vulnerability [Abelson et al., 1996a;
Abelson et al., 2001]. We have also shown that panic patients have abnormal HPA axis activity in a $24 \mathrm{hr}$ basal state study [Abelson and Curtis, 1996] and in a panicogenic laboratory model [Abelson et al., 1991]. In the original publications of these data, we speculated

Department of Psychiatry, Trauma, Stress and Anxiety Research Group, Drive, University of Michigan, Ann Arbor, Michigan

Contract grant sponsor: General Clinical Research Center grant; Contract grant number: MO1RR00042; Contract grant sponsor: National Institute of Mental Health; Contract grant number: RO1 $\mathrm{MH} 052724$.

*Correspondence to: Dr. James L. Abelson, 4250 Plymouth Road, Ann Arbor, MI 48109-5765. E-mail: jabelson@ umich.edu

Received for publication 29 August 2006; Revised 3 January 2007; Accepted 27 January 2007

DOI 10.1002/da.20317

Published online 7 June 2007 in Wiley InterScience (www. interscience.wiley.com).

This article is a US Government work and, as such, is in the public domain in the United States of America. 
that both the respiratory irregularity and HPA axis disturbances could be secondary phenomena and not primary factors in disease pathology [Abelson and Curtis, 1996; Abelson et al., 2001].

In a recent review of our panic-HPA work [Abelson et al., 2006], we suggest that HPA axis abnormalities in panic are secondary to hyper-reactivity to specific environmental triggers that are known to be highly salient to this system, such as novelty, and that HPA axis abnormalities in panic may thus originate in suprahypothalamic brain areas that modulate the HPA axis rather than within its core components. We have subsequently revisited our respiratory irregularity data [Abelson, 2006]. We had originally speculated that respiratory irregularity in panic might represent a secondary effort to use intrinsic anti-dyspneic effects of sighs to combat anxious distress associated with subjective dyspnea [Abelson et al., 2001]. Noting that (a) panic patients had elevated ACTH levels throughout our doxapram challenge study, relative to levels at the same time of day in a separate basal state study; (b) that they had elevated tidal volume irregularity and elevated sigh frequency relative to controls throughout the challenge study; and (c) that both ACTH and respiratory irregularity were unaffected by dramatic, drug-induced hyperventilation accompanied by panic attacks, we hypothesized that the elevated ACTH levels were marking a psychological-somatic hyper-reactivity to the novelty-induced stress of participating in a challenge study and that tidal volume irregularity could be a physiological reaction to the same sensitivity [Abelson, 2006]. If so, they should be significantly related to each other, and examination of this correlation (not previously done) could provide a very preliminary test of the hypothesis. We now present results of new correlational analyses examining the relationships between ACTH, tidal volume irregularity and symptom responses in the doxapram challenge study.

\section{METHODS}

The experiment compared 16 panic patients and 16 healthy controls on neuroendocrine, psychological and physiological responses to the respiratory stimulant doxapram, in a single session involving three phasesan accommodation phase $(5 \mathrm{~min})$, a placebo phase (12 $\mathrm{min})$, and a doxapram phase (25 min). Our primary prediction was that pre-challenge ACTH levels, which we thought best captured the HPA axis reactivity of subjects to the novelty stress of the challenge paradigm, would be strongly related to tidal volume irregularity and sigh frequency throughout the experiment. Pre-challenge ACTH was calculated as the mean of two samples taken during the accommodation and placebo phases, before doxapram injection. The injections were done out of sight of subjects, so subjective experiences during accommodation and placebo phases were identical, and the mean of these two samples provides the best measure of HPA activity during the first $15 \mathrm{~min}$ of data collection. Tidal volume irregularity was quantified by von Neumann's statistic [Abelson et al., 1996a] separately for the accommodation, placebo and doxapram phases. Subjects were recruited from a clinical population and through newspaper advertising, screened by structured diagnostic interview, and met strict entry criteria (including no daily medication for 2 months prior to study). All patients met DSM-IIIR criteria for panic disorder, with or without agoraphobia. For methodological details and overall results, see previous publications [Abelson et al., 1996a,b].

\section{RESULTS}

ACTH levels and tidal volume irregularity did not change significantly over the three phases and were elevated in panic patients relative to controls in all phases (see Table 1). Pre-challenge ACTH was strongly related to tidal volume irregularity during all three phases $(r=.67, P<.0001 ; r=.58, P=.0009$; $r=.63, P=.0002$, for accommodation, placebo and doxapram phases, respectively, $n=29$ ). It was also significantly related to sigh frequency during all phases (e.g., $r=.53, P=.006, n=25$ for total sighs, $r=.56$, $r=.002, n=29$ for sigh frequency after doxapram injection). To insure that these relationships were not a consequence of group differences on both variables, the relationships between pre-challenge ACTH and tidal volume irregularity during accommodation

TABLE 1. Mean (SD) ACTH and tidal volume irregularity over three phases in patients and control subjects, with repeated measures analysis of variance results

\begin{tabular}{|c|c|c|c|c|}
\hline \multirow[b]{2}{*}{ Phase } & \multicolumn{2}{|c|}{ ACTH $(\mathrm{pg} / \mathrm{ml})$} & \multicolumn{2}{|c|}{ Tidal volume irregularity } \\
\hline & Patients & Controls & Patients & Controls \\
\hline Accommodation & $32(20)$ & $17(5)$ & $517(422)$ & 175 (111) \\
\hline Placebo & $29(21)$ & $12(4)$ & $464(396)$ & $153(117)$ \\
\hline Doxapram & $34(16)$ & $16(7)$ & $495(339)$ & $192(101)$ \\
\hline ANOVA: $F(p)$ & & & & \\
\hline Group & \multicolumn{2}{|c|}{$7.1(.01)$} & \multicolumn{2}{|c|}{$11.2(.002)$} \\
\hline Phase & \multicolumn{2}{|c|}{$2.5(.10)$} & \multicolumn{2}{|c|}{$1.1(.33)$} \\
\hline Group $\times$ phase & \multicolumn{2}{|c|}{$1.7(.19)$} & \multicolumn{2}{|c|}{$0.3(.76)$} \\
\hline
\end{tabular}


and doxapram phases were examined for patients and controls separately. During accommodation, the relationship remained strong among patients alone $(r=.60, P=.02, n=15)$, but was lost among controls alone $(r=.06, P=.85, n=14)$. During doxapram phase, when all subjects were hyperventilating in response to drug, both groups appeared to contribute to the strength of the overall relationship, since the correlations were fairly robust and significant or nearly significant in each group, despite the small sample sizes in these secondary analyses $(r=.64, P=.01, n=14$ for controls; $r=.48, P=.07, n=15$ for patients).

There were some significant relationships between tidal volume irregularity and subjective distress or panic symptom intensity, but all of these relationships lost significance (all $P>.28$ ) when patients and controls were examined separately. When pre-challenge ACTH, subjective distress ratings, and panic symptom intensity ratings were entered into a multiple regression analysis predicting tidal volume irregularity, a substantial proportion of the irregularity variance was explained (46\% for accommodation phase, $40 \%$ for doxapram phase, $P<.001, n=29)$, but the distress and symptom variables made no meaningful contributions $(P>.73)$ beyond the variance explained by ACTH levels.

\section{DISCUSSION}

Pre-challenge ACTH levels and respiratory irregularity were strongly and consistently linked to each other in this study, whether irregularity was quantified by tidal volume variability or sigh frequency. ACTH explained nearly half the variance in tidal volume irregularity, and this link was independent of subjectively reported symptom and distress levels. The relationship between ACTH and respiratory irregularity was not examined in initial analyses because we had no theoretical reason at that time to expect that HPA axis activity and breathing patterns should be linked to each other. When prompted to look at this now by the realization that both could reflect sensitivity to contextual stress cues (e.g. novelty, anticipation of upcoming challenge), we were quite struck by the strength and robustness of the relationship we found in this data set. There is one prior report in the literature [Coplan et al., 1998] showing a parallel finding in a lactate challenge-elevated pre-challenge cortisol predicted greater fear, subjective dyspnea, lactate-induced panic, and hyperventilation (lower $\mathrm{pCO}_{2}$ ). Tidal volume irregularity and sighs were not reported, but our data would predict that their reduced $\mathrm{pCO}_{2}$ was due to more frequent sighs and that associated tidal volume irregularity would be strongly linked to pre-challenge cortisol. We hypothesize that this relationship appears primarily in the context of activation, as it was only absent in our data set for the control subjects during accommodation. We suspect that the control subjects were in a fairly basal state during accommodation but were activated by doxapram, whereas panic patients were activated in both phases due to hypersensitivity to paradigm novelty.

Though these post-hoc analyses can only provide the most preliminary kind of support for our hypothesis, it does suggest that follow-up work is indicated to directly test the proposal that both HPA axis activity and tidal volume irregularity (created by high rates of sighing) can be markers or consequences of hypersensitivity to factors such as novelty or anticipation of future challenge. Such work may help illuminate the neurobiology of panic disorder. This follow-up work must consider competing hypotheses as well, such as the possibility that HPA axis hormones themselves may directly modulate respiratory patterns.

\section{REFERENCES}

Abelson JL. 2006. Respiratory responses and respiratory irregularity in the Doxapram model of panic. Anxiety Disorders Association of America 26th National Conference on Anxiety Disorders. Miami, FL, March 24, 2006.

Abelson JL, Curtis GC. 1996. Hypothalamic-pituitary-adrenal axis activity in panic disorder: 24-hour secretion of corticotropin and cortisol. Arch Gen Psychiatry 53:323-331.

Abelson JL, Nesse RM, Vinik A. 1991. Stimulation of corticotropin release by pentagastrin in normal subjects and patients with panic disorder. Biol Psychiatry 29:1220-1223.

Abelson JL, Nesse RM, Weg JG, Curtis GC. 1996a. Respiratory physiology and anxiety: Cognitive intervention in the doxapram model of panic. Psychosom Med 58:302-313.

Abelson JL, Weg JG, Nesse RM, Curtis GC. 1996b. Neuroendocrine responses to laboratory panic: Cognitive intervention in the doxapram model. Psychoneuroendocrinology 21:375-390.

Abelson JL, Weg JG, Nesse RM, Curtis GC. 2001. Persistent respiratory irregularity in patients with panic disorder. Biol Psychiatry 49:588-595.

Abelson JL, Khan S, Liberzon I, Young EA. 2007. HPA axis activity in patients with panic disorder: Review and synthesis of four studies. Depression and Anxiety 24:66-76.

Coplan JD, Goetz R, Klein DF, Papp LA, Fyer AJ, Liebowitz MR, Davies SO, Gorman JM. 1998. Plasma cortisol concentrations preceding lactate-induced panic. Psychological, biochemical, and physiological correlates. Arch Gen Psychiatry 55:130-136.

Klein DF. 1993. False suffocation alarms, spontaneous panics, and related conditions. Arch Gen Psychiatry 50:306-317.

Papp LA, Martinez JM, Klein DF, Coplan JD, Norman RG, Cole R, de Jesus MJ, Ross D, Goetz R, Gorman JM. 1997. Respiratory psychophysiology of panic disorder: three respiratory challenges in 98 subjects. Am J Psychiatry 154:1557-1565.

Schreiber W, Lauer CJ, Krumrey K, Holsboer F, Krieg JC. 1996. Dysregulation of the hypothalamic-pituitary-adrenocortical system in panic disorder. Neuropsychopharmacology 15:7-15. 